\title{
Eine Provenzalische Bilderhandschrift.
}

Unter den Handschriften, welche die Buchhandlung Jacques Rosenthal in München, Karlsstrafse ro, zum Verkauf anbietet, fällt als besonders wertvoll ein in Südfrankreich geschriebenes Gebetbuch auf (vgl. Katalog VII Litterarische Seltenheiten. Bibliothekswerke N. 955). Herr Jacques Rosenthal hatte die Güte, die Handschrift auf einige Tage nach Halle zu senden, und uns so eine genauere Beschreibung $z u$ ermöglichen, als sie der Katalog bieten konnte. Die Veröffentlichung dieser Beschreibung wird gewifs den Freunden der mittelalterlichen Kunst willkommen sein. Denn so dringend auch zu wünschen wäre dals eine öffentliche Sammlung dieses kostbare Stück ankauft, liegt doch die Möglichkeit eben so nahe dafs es in den Besitz eines Privatmannes gelangt, und auf lange Zeit, ja vielleicht auf immer, unzugänglich wird.

Die Handschrift ist in rotes, mit Goldpressung verziertes Maroccoleder gebunden und trägt am Rücken, gleichfalls in Goldpressung: PSALTER | MSS. Sie besteht aus 255 Pergamentblättern (ungerechnet die beiden Deckblätter). Die Breite der Blätter ist 12 (bis $121 / 2$ ) cm, die Höhe 18 (bis $181 / 2$ ). Auf der ersten Seite des ersten Blattes steht von einer Hand des XVIJ.-XVIII. Jahrhunderts: Celestinorum S. Marcialis | De Gentillino fontis | Sorgensis. Wo mag dieses Kloster gelegen sein? Auf dem vorhergehenden Deckblatt der Handschrift steht mit Bleistift von ganz moderner Hand: Les Religieuses Trinitaires de Valence.

Von den 255 Blättern enthalten Bl. I-65 je ein Bild zum alten, Bl. 206-255 je ein Bild zum neuen Testament. Von den dazwischen stehenden 140 Blättern enthält $\mathrm{Bl} .66^{\mathrm{r}}-67^{\mathrm{r}}$ Stellen aus den Evangelien, $67^{\mathrm{v}}$ eine Ostertafel, $68^{\mathrm{r}}-73^{\mathrm{v}}$ einen Kalender. Dann folgt $\left(74^{\mathrm{r}}\right)$ der Vulgatatext des Psalters, der auf Blatt $172^{\mathrm{v}}$ zu Ende geht. Was dann folgt sind Cantica und Gebete, Alles Lateinisch. Ein kurzer Hymnus (Aurora iam spargit polum) ist etwa im XVII. Jahrhundert auf das Recto des Bl.. 206 geschrieben worden.

Besondre Beachtung verdienen der Kalender und die Ostertafel. Jener hat in seiner graphischen Einrichtung wie in seinem Inhalt grofse Aehnlichkeit mit dem von Suchier in den Denkmälern Provenzalischer Litteratur und Sprache I S. I IO-I 2 I abgedruckten Kalender. Auf eine bestimmte Lokalität kann aus den erwähnten Heiligen nicht geschlossen werden; doch weisen sie auf Languedoc im Allgemeinen hin.

Zeitschr. f rom. Phil. XXI. 
Der Name des 1274 gestorbenen Thomas Aquinas (Thome de ordine Predicatorum) ist zum 7. März erst nachträglich von andrer Hand eingesetzt worden. Wichtiger ist die Ostertafel, die das Datum des Osterfestes für die Jahre I 265 (mcc.lxv. d nonas april' u. s. w.) bis 1305 angiebt. Hiernach wird die Handschrift im Jahre 1264 angelegt worden sein. Dafs man damals die Jahreszahl bis zum Vorabend des Osterfestes auszudehnen pflegte, lälst die Eintragung Hic mutatur millesimus (im Kalender, $\mathrm{Bl} .69^{\mathrm{r}}$, zum 25. März) erkennen, die wohl mit Bezug auf den Tag von Christi Auferstehung (27. März, Resurrectio domini, Bl. 69 ${ }^{\mathrm{r}}$ ) an dieser Stelle steht.

Die Bilder sind in XIV. oder XV. Jahrhundert gezählt worden. Die Zahlen XX, XL, LX und LXVI, welche auf dem Verso von Bl. 19, 39, 59, 65 stehen, zeigen dafs unter den Bildern vor Bl. 19 ein Bild fehlt: offenbar das zwischen Blatt 6 und 7 ausgeschnittene Bild. In der Bilderreihe des neuen Testaments hat der Zähler Bl. 224 mit XX, Bl. 24 I mit XL, Bl. 225 mit LVI bezeichnet; folglich fehlen: ein Bild vor Bild XX, drei Bilder zwischen $\mathrm{XX}$ und $\mathrm{XL}$ und zwei Bilder zwischen XL und LVI, und zwar ist ein Bild ausgeschnitten zwischen $\mathrm{Bl} .207$ und 208, zwei zwischen 234 und 235, eins zwischen 240 und 24 I, eins zwischen 246 und 247; der Platz des sechsten Bildes, das fehlt, lälst sich nicht genau bestimmen.

Es folge nun die von Kautzsch verfalste Beschreibung der Bilder, und auf diese Suchiers Abdruck der Provenzalischen Unterschriften. Einige Stellen (I I. 21. 22. 25. 33. 101. 106) bleiben offen, da sie von einem Benutzer durch Auftragen chemischer Stoffe entstellt sind. Die Zahl der zur Ausfüllung dienenden Punkte ist im Druck so gewählt dafs einem jeden Punkt in der Handschrift der Raum eines halben $\mathrm{cm}$ entspricht. Im Text sind grolse Buchstaben und Apostrophe gesetzt, doch sind die Punkte der Handschrift beibehalten; sonst ist nichts an der Ueberlieferung geändert. ${ }^{1}$ Buchstaben, die einer aufgelösten Abkürzung entsprechen, sind kursiv gedruckt.

Die Handschrift zerfällt, wie aus der Beschreibung hervorgeht, in drei Teile. Auf Blatt $\mathrm{I}-65$ finden sich nur Bilder mit provenzalischen (und beträchtlich jüngeren lateinischen ${ }^{2}$ ) Unterschriften. Diese Bilder messen durchschnittlich $8(8,5) \times 12(12,5) \mathrm{cm}$ und nehmen die ganze Seite ein. Mit Blatt 66 a beginnt sodann der laufende Text, der nur mit Randverzierungen und Bilderinitialen geschmückt ist. Dieser Teil des Schmucks ist von anderer Hand als die grofsen Bilder vorn. Dagegen setzt der erste Maler wieder ein bei der Fortsetzung des grofsen Bilderkreises nach Schlufs des

1 Nur $7 c^{\prime}$, das am Schlufs vieler Unterschriften (von 8 an) steht, ist im Druck weggelassen.

2 Von diesen im XVI. oder XVII. Jahrhundert eingetragenen Lateinischen Unterschriften wird, da sie keinerlei Interesse bieten, in unserer Beschreibung ganz abgesehen. 
Textes: noch einmal 50 Bilder auf ebenso vielen Blättern folgen und beschliefsen das Ganze.

Die grofsen Bilder vorn und am Ende entsprechen einander in der äufseren Behandlung genau. Auch inhaltlich gehören sie untrennbar zusammen. Der Bilderkreis beginnt mit Darstellungen zur Schöpfungsgeschichte. Es folgen die Erlebnisse des ersten Elternpaares und ihrer Söhne, die Geschichte Noahs, der Turmbau zu Babel, Isaaks Opferung und das bekannte Bild: Abrahams Schols. Hierauf sofort (ohne dafs eine Scene der sonst so beliebten Josephgeschichte illustriert wäre) Bilder zum zweiten Buch Mose bis zur Bestrafung des Volks nach den Vorgängen am Sinai. Sodann Illustrationen zur Geschichte Davids von seinem ersten Erscheinen vor Saul bis zum Tod Absaloms. Auffallenderweise folgt hier ein Bild, wie Simson den Löwen tötet. Die beiden Darstellungen: Salomos Urteil und sein Götzendienst beendigen die Bilderreihe zum Alten Testament. Ohne weiteres schliefsen sich Darstellungen zur Geschichte des Elternpaars der Maria und zu ihren eigenen Erlebnissen an bis zur Vermählung. Sehr befremdlich schliefst hierauf ein Bild des Glücksrads die erste Hälfte. Es folgt der Text, und nach dessen Schlufs geht die Bilderfolge unmittelbar weiter mit der Verkündigung an Maria. Die Jugendgeschichte Jesu ist ausführlich illustriert bis zur Taufe im Jordan. Auf die Auferweckung des Lazarus und die Versuchung Jesu folgt dann sofort die Passionsgeschichte vom Einzug in Jerusalem bis zur Himmelfahrt. Pfingstfest, Tod, Begräbnis und Krönung Mariä, Jüngstes Gericht, Christus in der Glorie, endlich der Erzengel Michael machen den Beschlurs.

In dieser Bilderreihe, so wie sie ist, fallen die Einschiebsel Abrahams Schols und das Glücksrad auf. Andererseits fehlen Scenen der biblischen Geschichte, die man erwarten könnte. Auch Abweichungen von der üblichen Reihenfolge der Bilder finden sich. Darnach scheint die Bilderreihe nicht original, sondern eine freiere Redaktion einer älteren Vorlage zu sein. Die ursprüngliche Reihenfolge dürfte sich nicht überall mit voller Sicherheit herstellen lassen. Doch gehört offenbar das 15 . Bild (Bl. $15^{\mathrm{r}}$ ) unmittelbar hinter das 10. (Bl. 10 $)$ als dessen Gegenstück.

Die Bilder sind durchweg in Deckfarben auf Goldgrund gemalt und von farbigen Rahmen umschlossen. Diese Rahmen zeigen ziemlich breite Leisten. Sie sind blau und rot gemalt und zwar so, dafs immer die einander gegenüberstehenden Leisten die selbe Farbe haben. Innerhalb der Bilderfolge wird nun abgewechselt: sind beim ersten Bild Ober- und Unterleiste rot, so zeigen beim zweiten Bild die Seitenleisten diese Farbe, während dort die Seitenleisten, hier Ober- und Unterleiste blau sind. Auf den farbigen Grund der Leisten sind mit weifser Deckfarbe Ornamente gemalt. Auf die quadratischen Eckfelder der Rahmen ist Gold aufgelegt. Um den ganzen Rahmen ist in einigem Abstand eine schwarze Linie gezogen, der Zwischenraum ist unbemalt geblieben. 
In den Bildern findet sich, von unten nach oben gerechnet, zunächst regelmärsig eine braune Bodenwelle. Darüber dehnt sich der Goldgrund aus. Das Gold ist, wie üblich, auf eine feine weilse Masse aufgetragen und poliert. Auffallenderweise füllt der Goldgrund meist nicht den ganzen Raum innerhalb der Rahmen. Er schliefst vielmehr nach oben kleeblattförmig ab, so dafs nur das mittlere Blatt die Oberleiste des Rahmens erreicht, während in beiden Ecken Winkel frei bleiben. Diese sind mit ziegelroter (ganz vereinzelt auch grüner) Farbe bemalt, und auf diesem Grund ist mit weifser Deckfarbe die Zeichnung einer Backsteinmauer angebracht. Diese vergoldete und bemalte Fläche bildet den Hintergrund der Darstellungen. Von Landschaft ist natürlich keine Rede. Die übliche Bodenwelle und hie und da ein paar stilisierte Bäume genügen. Diese haben durchweg eine und die selbe Form: kurzen Stamm, aus dem ein bis drei schlanke Stengel aufwachsen, die oben $j e$ in ein rundes Blatt mit verziertem Bogenrand auslaufen. Reben und brennender Busch sind übrigens von den Bäumen unterschieden, aber ebensowenig naturalistisch. Für die Architektur treten Bogen ein, die von einer Rahmenleiste zur anderen gehen, sodann Thore oder ein irgendwie gefärbter Grund statt des Goldgrunds - soweit sie überhaupt angedeutet ist. Selbst das Gerät ist nur soweit irgend nötig, nirgends mit geflissentlicher Ausführlichkeit angegeben.

Die Menschen nehmen etwa $4 / 5$ der Höhe der Bildffäche ein. Sie sind nicht streng stilisiert. Dies aber nicht etwa, weil der Maler seiner Zeit voraus wäre, sondern weil er offenbar ein Routinier ist, dem es nur auf die Masse des Geschaffenen, nicht auf dessen Vollendung ankommt. Von der ursprünglichen Vorzeichnung ist nichts mehr zu erkennen, da die Farbe überall deckt. Diese ist zunächst in einem gleichmälsigen Ton über die ganze Fläche ausgebreitet. Darauf sind dann in einem dunkleren Ton der selben Farbe die Schatten aufgemalt. Endlich sind Umrisse und Innenzeichnung mit dem Pinsel in ziemlich starken schwarzen oder braunen.Linien nachgezogen. So fehlt der Zeichnung jedes individuelle Leben. Wir haben weder einheitliche Typen noch feinere Charakteristik. Die Figuren folgen nur der gewohnheitsmäfsig ausgebildeten Schablone ihres Verfertigers. Ueber die Gesichter wären etwa noch ein paar Worte zu sagen. Sie sind (wie die Hände) nicht ausgespart, sondern zunächst mit Weifs gedeckt. Darauf sind in Rosa die Schatten gemalt, endlich ist die Zeichnung mit ziemlich spitzem Pinsel in Braun oder auch Rot eingetragen. Die Lippen sind stets durch einen roten Strich ausgezeichnet. Das Haar ist durchweg blond, bald dunkler, bald heller gelb. Von Gesichtsausdruck kann man nicht sprechen, alles Innenleben ist in wenigen stereotypen Handbewegungen dargestellt. Ebenso verraten Haltung und Stellung, Handlung und Erlebnis durchaus keine selbständige frische Beobachtung.

Die Farbenskala zeigt Purpurrot (stumpf), Ziegelrot, Rotbraun, brandiges Rosa, Gelb, Hellgrün, Dunkelblau (stumpf), Himmelblau, Weils, Schwarz. Sämtliche Farben decken. 
Wir müssen nach alledem sagen, dafs diese Miniaturen keineswegs auf einer hohen Stufe stehen: sie tragen den Stempel gewerbsmärsiger Arbeit. Aber gerade darin liegt auch wieder ihr eigentümlicher Wert. Ihr Charakter lälst auf eine einstige weitere Verbreitung dieses Bilderkreises schliefsen, dessen einziges erhaltenes Exemplar sie zu bilden scheinen.

Von der Reihe der grofsen Bilder unterscheidet sich zu seinem Vorteil der Schmuck des eingeschobenen Textes. Zwar die drei ornamentalen Leisten in Gold, Rot, Gelb und Blau, die sich jedesmal links auf den drei ersten Textseiten finden, stehen nicht eben hoch. Dagegen sind die Drolerieen auf dem äufseren (rechten) Seitenrand jeder Recto-Seite nicht übel. Es ist jedesmal eine einzelne Figur, die nach unten in einen langen Schwanz endet, so dafs das Ganze als Seitenleiste dienen kann. Die Zeichnung dieser Stücke ist sicherer und im Stil strenger (wir haben die bekannten Kopftypen mit kleinen gerundeten Nasen und offenen, ziemlich gewölbten Augen) als in den grofsen Bildern. Die Erfindung ist frisch, wenn auch ohne den übersprudelnden Humor, der so manche andere Drolerieen auszeichnet.

Endlich finden sich auch Initialen. So beginnt jede Kalenderseite mit einem gröfseren $K l$ in Blau, Rosa und Gold. Weiter stehen zu Anfang der einzelnen Textabschnitte gröfsere rote oder blaue Buchstaben, die mit Federstrichranken in blauer, roter oder violetter Tinte umsponnen sind. Schliefslich fehlen auch grofse Bilderinitialen nicht. Diese sind derart ausgeführt: Buchstabenkörper und rechteckige Umrahmung sind in Blau und Rot gehalten und mit weifsen Ornamenten verziert. Beim ersten Initial ist noch eine schmale goldene Leiste um das Ganze gezogen. Der Grund innerhalb der Buchstaben ist mit Gold ausgelegt. Auf diesem Grund stehen die Figuren. Diese sind mit Deckfarben ganz in der Technik der grofsen Bilder vor und hinter dem Text gemalt, aber von der Hand des Malers der Drolerieen, mit denen sie stilistisch genau übereinstimmen.

$[B l . \mathrm{I} v] 1$.

Lo primjer iorn que dieus comenset a crear | lo mon e ueus com tenc lo mon e som poder.

[2r] 2 .

El segon iorn com dieus deuezi la terra de las aiguas.

[3v] 3 .

El ters iorn com dieus mes lo soleil e la luna | e las estelas el cel.

[4r] 4 .

El quart iorn com dieus creet los arbres.

$\left[5^{\mathrm{v}}\right] 5$.

Com nostre seinher al sinquen dia creet la [sic] bestias / els aucels els peissons de la mar. 
[6r] 6.

Com nostre seinher cant ac fag home e l'ac fag adormir | trais li una costa del destre costat e fes ne femena ad / aiutori d'el. Al seizen jorn.

\section{[7 $\mathrm{r}] 7$.}

Com nostre seinher mostra l'albre ad Azam $e$ ad Eue [sic] / cant los ac mes em paradis e ueda ad els que non majessont [sic] del frug d'aquel albre.

$[8 \mathrm{v}] 8$.

Com Azam e Azeua maieron [sic] del pom de l'albre / que dieus lur auia deuedat per amonestament | del serpen so es assaber lo diable.

[9r] 9.

Com nostre seinher uenc dauan Azam e Azeua | et els de uergonha que agron cant si uiron nutz | cobriron lur natura cascun d'una fuella d'albre | e demandet per que auion maniat del frug que el lur auia/ uedat. e Adam respos Seinher la femna que m'as dada o a / fag far.

[rov] 10.

Com nostre seinher fes gitar Azam $e$ Azeua | a l'angeł car li foron dezobedien de paradis.

[IIv] II.

Com Azeua jac de dos fils bessos, que l'us ac nom / Abel e l'autre Cahim e foron los prumiers effan (?) | que . . . . .

\section{[1 $12 \mathrm{r}] 12$.}

Com Cahim e Abel que eron fraires sacrificauon | a nostre seinhor de lur blat . et Abel sacrifiaua / del mellor que auia. e Cahim del auollor que auia.

$$
\text { [13v] } 13 .
$$

Com Cahim aucis son fraire Abel per enueia.

$$
\text { [14 r] } 14 .
$$

Com nostre seinher parlet a Cahim cant ac | mort son fraire Abel . e demandet li On es tos | fraire Abel .e el respos. E soi eu garda de mo fraire.

$$
\left[15^{r}\right] 15 \text {. }
$$

Com Azam e Azeua comenceron a trebaillar | cant foront gitat de paradis.

$$
[16 \mathrm{v}] 16 .
$$

Com nostre seinher mandet per l'angel a Noe que fezes l'arca | en que gandis aquelas cauzas qu'el li mande cant | uolc destrure lo mon per aigua per la malicia de / las gens.

$$
[17 \mathrm{r}] \mathrm{i} 7 .
$$

Cant Noe ac facha l'arca que dieus li ac mandat / cant saup que lesduluuis deuia uenir. e com mes | de cascuna creatura un pareil en l'arca.e aquellas | personas que dieus li ac mandat.

$$
[18 \mathrm{v}] 18 .
$$

Com l'arca anaua per l'esduluui de las aiguas e com | totas cauzas periron estier aquo que se saluet en / l'arca dauant dicha.

8. Wegen Azeva, aus na Eva mit eingeschobenem z, vgl. Suchier, Denkmäler Prov. Litt. I S. $\mathbf{5 7 2 .}$

I6. mandet] $\mathrm{t}$ steht über der Zeile, weil vom Schreiber nachträglich hinzugefügt. Das zweite mande blieb ungeändert.

18. aiguas] i ist von einer spätern Hand in y geändert. 
[19r] 19.

Cant l'esdolobis fon passatz e remas l'arca entre dos | puegz que son en Erminia segon que hom dis.e com / Noe cant conoc que las aiguas eron amermadas trames / lo corp per uezer se las aiguas eron baizadas [sic] e el trobet | una caronhada e comenset a maniar e non tornet. | e pueis el trames la columba que li aportet i. ram d'oliuer $\mid e$ issiron de l'arca.

[20v] 20.

Com Noe coil los razims de la uinha que auia / plantada . e fon lo prumiers hom que plantet | uinha.

$$
\text { [2I r] } 21 \text {. }
$$

Com Noe estet cant fon ibres(?) del uin que ac begut (?) de / la uinha que auia plantada . e com l'us de sos fils lo / descobri . . . . . . . .

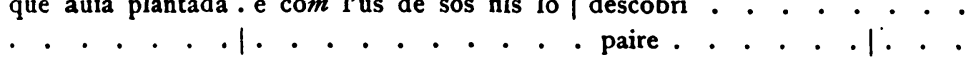

$$
[22 \mathrm{v}] 22 .
$$

Com le [sic] gentil bastian la torre la cal es appellada la | Tor Babel la cal es em Babulonia . . . . . . . . . . . . . . . . . . . . . . . . . . . . . . . . . . . . . .

$$
\left[23^{\mathrm{r}}\right]^{23} \text {. }
$$

Com Abraam uolc sacrificar son fil Ysaac a nostre / seinhor car el li o auia mandat. e cant ui sa uoluntat / trames li son angel que li o uedet e mostret li .i. mouto / de que feses sacrifizi a lui.

$$
\left[24^{v}\right] 24 \text {. }
$$

Com Abraam ten las armas en son sen.

$$
[25 \mathrm{r}] 25 .
$$

Com nostre seinher parla a Moysen . . . I anes a Pharaon . . . . . . . . I et ades auia . . . . . . . . / Moysen. . . . non... .

$$
\text { [26v] } 26 .
$$

Com Moysen se merauillha [sic] cant ui lo boisson / uert que dauant l'era ueiaire que cremes cant nos-|tre seinher parlaua ab lui.

$$
\text { [27 } 7^{x} 27 .
$$

Com Moysen dis a Pharaon que dieus li manda que el / desliure som pobol.

$$
\text { [28v] } 28 .
$$

Com Moysen desliuret lo pobol d'Israhel per la | uoluntat de dieu del poder de Pharao . que non l'auia uol-Igut alargar per neguna pestilencia que dieus li agues / tramessa denant.

$$
\text { [29r] } 29 .
$$

E com la mar si obri pel tocamen de la uerga de / Moysen.per la uoluntat de dieu . e enaissi passet | outra ab tot lo pobol el dezert.

$$
\text { [30v. } 31 \mathrm{r} \text { r] 30. } 31 \text {. }
$$

Com Faraon peri en mar ab sa caualgada que seguia [31 $\mathrm{r}$ ] lo pobol que Moysen ne menaua qu'el cuiaua [30v] far tornar areires e tener e som poder.

30. 31. Die beiden Bilder haben nur eine, über beide Seiten laufende Unterschrift. 


\section{[32v] 32.}

Com nostre seinher trametia la marna cascun / iorn al pobol cant foron el desert e issit del poder / de Pharaon. de que uinian.e aquella manna | auia lur sabor de cal que uianda aguesson talen / e duret lur quaranta ans. e perderon o per lur follia.

\section{[33 r $] 33$.}

Com Moysen monstra la serpe al pobol que ac facha / per mandamen de dieu que alcunas serpens poinhion | alcuns del pobol e morion per aquella poinchura . e | el di lur que cant neguns seria poingz d'aquellas serpens / gardesson aquella e serion guerit. | Et aquella serpens signifiquet nostre senhor que deuia esser | mes en cros per l'uman linhatgue . . . . . . . . . . la passion... .

$$
\left[34^{\mathrm{v}}\right] 34
$$

Com nostre seinher dona la lei a Moysen el pueg de / Synai e e la neuol qu'es entr'amdos. et aquella leis / era escricha en taulas de.

\section{[35 r] 35 .}

Com lo pobols esta el dezert entre tan que Moysen anet / recebre la lei de nostre seinhor. e estet $i$ per .xl. iorns | e .xl. nuegz.

$$
\text { [36v] } 36 .
$$

Com alcuns del pobol adoron lo uedel que / agron fag entre tan que Moysen esperaua la lei . que / dieus li donet.

\section{[37 r] 37.}

Com Moysen di al pobol que dieus li a donada la lei | en taulas.

$$
\text { [38v] } 38 \text {. }
$$

Com Moysen a triatz aquels que non adoreron lo | uedel ni mescrezeron lọ nostre seinhor.

$$
\text { [39 r] } 39 .
$$

Com Moysen fa aucir aquels que agron peccat en adorar $\mid \cdot l o$ uedel $e$ desconogut dieu . e fes aucir los us als autres.

$$
\text { [40v] } 40 .
$$

Com Dauid que era pastre uenc dauant Saul que era reis $e$ / oingz de dieu e Saul mostra a Dauid sa filla Nicola / e dis li que dara la li per moller . si aucis Golias lo / iaian qu'es campios contra el e contra som pobol.

$$
\text { [4I } \mathrm{r}^{\mathrm{r}} \mathbf{4}^{\mathrm{I}} \text {. }
$$

Com Dauid aucis Golians ab i. peira de fonda.e pueis | tolc li lo cap.e aisso fes per la uoluntat de dieu.

$$
\text { [42v] } 42 \text {. }
$$

Com Saul dona sa filla a Dauid car a mort Golias e moutz d'autres de sos enemics.

$$
\left[43^{r}\right] 43 .
$$

Com la femna encantairis que sabia de l'art de nigromancia / fes cemblant a Saul quel fezes parlar ab Samuel la propheta| pueis que fon mortz.

33. Die allegorische Deutung beruht auf Foh. III. 14. Die letste Zeile ist am untern Rand bis auf geringe Spuren vom Buchbinder abgeschnitten. 
car Saul si cosseillaua ab el entre tan / que uiuia . que uolial demandar com li penria de la batailla / que deuia far ab los Faristieus.

$$
\text { [44 } \left.{ }^{\mathrm{v}}\right] 44 \text {. }
$$

Com Saul aucis se mezeis per dolor que ac cant ui que | sos enemics uencion sas gens. e com sos escudiers / lo soste cant ui que moria.

$$
\text { [45 } \left.{ }^{\mathrm{r}}\right] 45 \text {. }
$$

Com Dauid bailla las letras ad Urias son caualier que las bail a de so | senescalc que estaua per lui en la batailla contra sos ene/mics en las cals letras si contenia que el meseis que por/taua las letras fos mes en tal luec de batailla que moris. | Car Dauid auia emprenhada sa moller e no uolia que o saubes el.

[46v] 46.

Com la propheta reprent Dauid per lo peccat que / auia fag que era grans d'Urias e de sa moller.

[47 r] 47.

Com Dauid si soterret per lo peccat que auia fag | per so que nostre seinher loil perdonæs cant la propheta / l'en ac repres.e adonc fes los set salmes e nostre / seinher trames li aqui son angel.

$$
\text { [48v] } 48 .
$$

Com Absalon fil de Dauid que era lo plus bels hom del / mon que guerrejaua ab som paire cant uenc .i. iorn | que fugia per aisso que la ost de som paire uencia la soa. | et en .i. bosc remas pendutz en .i. albre e son caual | emblet si desotz el. et us cauallier de som paire / que l'encausaua aucis lo.

$$
\text { [49 r] } 49 .
$$

Com Sanson fil de Dauid [sic] que era lo plus fortz hom del mon . I que pueis mori per sa forsa. tolc per forsa la bresca al leon | quen portaua.

$$
\text { [50 v } 50 .
$$

Com Salamon fil de Dauid que fon lo plus sauis hom | del mon jutguet lo contrast de l'efan que era/entre las doas macipas aissi com si conten en la pis/tola.

$$
\text { [51 r }] 51 \text {. }
$$

Com Salamon adoret los dieus de la pagana per / amor d'ela tan la amaua . en dezamparet lo sieu dieu / uer.e cant si reconoc d'aquel fallimen que auia fag / contra dieu fugi s'en en i. bosc.

$$
\text { [52v] } 52 \text {. }
$$

Com Joachim e Anna paire e maire de nostra / dona foron acomiadat del temple per lo capela | del temple. e no uolc penre lur offerta. Car escrig / era en la lei uiella que qui non auia efan era mal/dig de dieu e els no' $n$ auian minga $e$ auion estat ganre esems.

\section{[53 $\mathrm{r}] 53$.}

Com l'angels conortet sancta Anna cant si marria / en l'ort . e dis li qu'ela era preins d'un efan que anc mais | non fo som par ni sera. el cal ort era ab sa siruenta / pres d'un laurier.

43. Faristieus] gemeint sind die Philister. Der Schreiber hat woohl an die Pharisäer gedacht.

49. Das fil de Dauid ist aus der vorhergehenden oder folgenden Überschrift eingedrungen. 


\section{[54 v] 54.}

Com l'angles [sic] uenc a Iohachim qu'estaua el desert | maritz ab sos pastors $e$ ab som bestiari e dis li que | sa moiller era preingz d'una filla e que tornes ad | ella $E$ era cosseupuda enans que fossetz acomiadat | del temple e car tu non o sapias e tu lauias dezampa|rada.

\section{[55 r 55 .}

Com Iohachim pres l'anhel de que auia couidat l'angel | de maniar.e preguet a nostre seinhor qu'el deinhes rece/bre son sagrifizi . car l'angels li o ac aissi dig.

$[56 \mathrm{v}] 56$.

Com l'angels uenc a Ioachim cant dormia . e dis | li co non tornaua a sa moller Anna aissi com el li | auia dig.

\section{$\left[57^{\mathrm{r}}\right] 57$.}

Com Ioachim parla ab los pastors sieus cant / l'agron leuat del sol o era ablesmatz que si cuiauon | que fos mortz. et el comta lur so que l'angels li ac dig.

$$
\left[5^{8 v}\right] 58 .
$$

Com Ioachim e sancta Anna s'encontreron a Portas | Aurias e s'abrasseron aissi com l'angels lur o ac $\mid$ dig.

$$
\text { [59 r] } 59 .
$$

Com sancta Anna jas de nostra dona.

$$
\text { [60v] } 60 .
$$

Com Ioachim e sancta Anna ameneron nostra|dona lur filla al temple. [6r r 61 .

Com Iohachim e sancta Anna s'en tornon a lur maizon / cant agron laissada nostra dona al temple per seruir.

$$
[62 \mathrm{v}] 62 .
$$

Com Habiatar e Ioachim e sancta Anna e nostra dona / pregauon nostre seinhor que lur demostres cals | deuia esser espos d'ella.

$$
\left[63^{r}\right] 63 .
$$

Cant la trips de Iudas ac gazanhada la sort de las | autras trips. e pueis aquella trips de Iudas aiustet si / e cada uns tenc sa uerga el man e pregauon a nostre seinhor / que lur deinhes demostrar alcun signe en la uerga d'aquel | que uolria que fos espos de Maria. E com adoncs uenc la collumba en la uerga de Ioseph . e era plus vieils que negus | dels autres.e aquel fon espos de Maria.

$$
\left[64^{v}\right] 64 \text {. }
$$

Com Iosep en mena Maria e sa maizon ab las / uerges queil fazessont companhia.

$$
\left[65^{\mathrm{r}}\right] 65 \text {. }
$$

Aisso es auentura so es assaber la roda del mon.

[206v] 66.

Com l'angels Gabriel anunciet a nostra dona / cant li dis Aue Maria.

$$
\text { [207r] } 67 .
$$

Com Josep si merauillet cant ui nostra dona espoza | sieua preinh . que no sabia com era uengut . e rep|taua ne las uerges a cui l'auia comandada.

$$
\text { [208v] } 68 .
$$

Com sọ nostra dona efantet e jac de nostre / seinhor. 
[209r] 69.

Com anossiet l'angels als pastors que nostre seinher / era natz em Beleem.

$$
\text { [210v] } 70 .
$$

Com nostre seinher fon circumcis . a l'octau jorn de sa | natiujtat . e fon apellatz Jezus.

$$
\text { [21 } 1 \text { v] } 71 \text {. }
$$

Com li tres rei uengron dauant Erodes que anauont | adorar nostre seinhor. E Erodes preguet lur que / cant l'aurjont trobat que tornessont ad el $e$ que loil | esseinhessont que el l'iria adorar.

$$
\text { [212 r] } 72 .
$$

Com li tres rej adoreront nostre seinhor e li offriron | aur. e ensens. e mirra.

$$
\text { [213v] } 73 .
$$

Com nostra dona offri nostre seinhor el temple / a saing Symeon . ab doas columbas.

$$
\text { [214v] } 74 \text {. }
$$

Com l'angels conortet Josep que s'en uolia fugir / d'ira e de uergoinha cant ui nostra dona sa espoza / preinh . e l'angels dis li que del saing esperit auia | coceuput.

$$
\text { [215 r } 75 .
$$

Cant l'angel dis als reis que agron adorat nostre seinhor / que non tornesson ad Erodes.

$$
[216 \mathrm{v}] 76 .
$$

Com Jozep e nostra dona s'en fugion ab nostre seinhor/per paor d'Erodes en Egipte. Que l'angels o ac reuelat la nueg a Iozep.

$$
[217 \mathrm{v}] 77 \text {. }
$$

Com Erodes comandet a sos caualiers aucire los enfans | totz de son regne que foron .c.xliiii. milia.

$$
[218 \mathrm{r}] 78 .
$$

Com Erodes fes aucir los enfans que foron .cxliiii. milia.

$$
\text { [219r] } 79 .
$$

Com nostra dona e Iozep troberon nostre seinhor cant / l'agron perdut el temple ques desputaua ab los Juzieus.

$$
\text { [220v] } 80 \text {. }
$$

Com saingz Jo. babtista batejet nostre seinhor $e$ adonc auja .xxx. ans.

$$
\text { [22IV] } 81 \text {. }
$$

Com nostre seinher ressuscitet lo Lazer pels precs / de sancta Marta e de la Magdalena serors d'el.

$$
\text { [222 } \mathrm{r}] \mathbf{8 2} \text {. }
$$

Com lo diables uolc temptar nostre seinhor cant | fazia la quarantena el desert. que li dis que Se / fils de dieu iest digas que aquestas peiras siant pas / e mangja ne.e el respos li. Escrig es que hom no uiu / de sol pan. $\mathrm{E}$ pueis portet lo sobrel temple e dis li ques / gites a ual.

\section{3. saing] $g$ ist in $t$ verwandelt.}

74. coceuput] $\mathrm{u}$ ist später eingefügt in coceput.

80. Jo.] ist über der Zeile nachgetragen. 
Com nostre seinher jntret en Jerusalem caualgant | en la sauma.e la honor queil fil dels Ebrieus li fazion.

$$
\text { [224 r }] 84 \text {. }
$$

Com nostre seinher lauet los pes als apostols la | nueg de la cena.e dis lur Essemple uos don que | enaissi com eu o fauc enaissi o fassatz uos autre.

[225 r 85 .

Com Judas pren los trenta deners per los cals ac donat / e uendut nostre seinhor. e pueis los lur rendet.

$$
\text { [226v] } 86 .
$$

Com nostre seinher cenet ab sos apostols | e com saingz Johans si enclinet son cap sobrel | pieg de nostre seinhor.

$$
\text { [227v] } 87 \text {. }
$$

Com li Juzieu prezeron nostre seinhor cant Iudas | lo lur ac uendut.e Iudas lur ac dig aquel qu'eu bai|zarai prenes . e ueus col baiza.

$$
\text { [228 r } 88 .
$$

Cant li Juzieu agron pres nostre seinhor com | l'adusseront dauant Pilat.

$$
\text { [229 r] } 89 .
$$

Com li Juzieu cant agron pres nostre seinhor / ni adug en la carcer que per isquern li uestiron .i. | pali e l'asegron en la cadieira el mezeron una uerga | el man. et agenolanon si denant el . per esquiern / eil donauon a la gauta . e disseron li Dieus ti sal rei $\mid$ dels Iuzieus.

$$
\text { [230v] } 90 .
$$

Com saing Peire si ploret cant ac negat nostre / seinhor . e recordet si cant auzi lo gal cantar. per la | paraula que nostre seinher li auia dicha. so es assaber que | Auans quel gals cante mj auras negat iii. uegadas.

$$
\text { [231r] } 91 \text {. }
$$

Com nostre seinher es dauant Erodes que Pilatz loil $\mid$ ac trames per uezer. Car Erodes loi auia mandat e pregat / e per amor d'aisso Pilatz e Erodes foron amic . que si uolgron (?) $\mid \mathrm{mal}$.

$$
\text { [232v] } 92 .
$$

Cant lo rei Erodes fes tornar nostre sejnhor cant l'ac | uist a Pilat . e l'ac fag uestir de blanc car lo tenc per fol | que noil uolc parlar.

$$
\text { [233v] } 93 \text {. }
$$

Com Pilatz fes batre nostre seinhor.

$$
\text { [234 } \left.{ }^{\mathrm{v}}\right] \text { 94. }
$$

Com Pilatz lauetz sas mas cant ac liurat | nostre seinhor al [sic] Juzieus a crucificar .e dis lur | Eu no soi nosens del sanc d'aquest drechurier.

$$
\text { [235 } \mathrm{v} \text { ] } 95 .
$$

Dels Juzieus com deuiziront la rauba de nostre / seinhor per sortz que i pauzeront cant l'agron / mes en cros.

\section{[236r] 96.}

Com Judas si pendet per dezesperansa car ac | uendut nostre seinhor.

85. Hinter pueis steht senpes e (durchgestrichen).

87. Iudas] beide Male stand Iuzas, $z$ in d geändert. Ebenso 96.

90. li auia] anfangs stand lauia.

94. lauetz] über dies geschwänzte $\mathrm{t}$, das ganz wie $\mathrm{tz}$ aussieht, vgl. Suchier, Denkmäler I S.481-2. 
[237 $\mathrm{v}$ ] 97.

Com dessenderont nostre seinhor de la cros.

[238 r] 98.

Com oiceront las plagas a nostre seinhor / e com fon pauzatz el monumen.

[239v] 99.

De la ressurexio e com nostre seinher ressucitet.

[240v] 100.

Com las tres Marias uengron al monumen en que / nostre seinher fon pauzatz . e l'angels dis lur dịs que | ressucitatz era e non era aqui.

[241 r] IOI.

Com nostre seinher aparec a la Magdalena / cant fon ressucitatz e dis li Nom uuelas tocar. | E dis li (?) . . . als apostols e a Peire qu'en | Galileam: ueiran (?) aissi com eu lur auia dig.

$[242 v] 102$.

De nostre seinhor cant mostret sas plagas a saing Thomas.

[243 '] 103.

Com nostre seinher s'en pujet el cel . uezent | sa maire e uezen sos apostols. [244v] 104 .

Com nostre seinher trames lo saing esperit als apostols. | els alumenet de la sua gracia . $e$ aqui mezeis sau|pron parlar de totz lengatgues.

[245 r 105.

Del passamen de nostra dona que nostre seinher pres s'arma.

[246v] 106.

Com saing Peire e saingz Pauls porteron nostra | dona sebelir cant fon passada . e com li Iuzieu la / lur cujauon tolre rema . . . . . . . .

$\left[247^{r}\right] 107$.

De nostre seinhor com coronet nostra dona maire de lui.

$[248 \mathrm{v}] 108$.

Com nostre seinher uenra iutgar al iorn del iuzizi |. Com nostra dona e saing Johans pregaran per lo huma linatgue.

[249 r] 109.

Com las gens ressucitarant al iorn del iutgament | els angels com cornarant. [250 r] 110.

Com Abraam menara los iustz en gloria | apres lo jutgamen.

[251 v] 111 .

Com las gens istarant em paradis.

[252v] 112.

Com lo diables en menara los peccadors en efern / cant nostre seinher aura donada la sentencia al iorn / del juzjzi.

[253 r] 113.

Dels peccadors com estarant en efern.

[254v] II 4 .

Com nostre seinhor esta en sa magestat.

[255 r] 115.

Saing Michel archangel com aucis lo colobre.

I10. Hinter Abraam ist ne wegradiert.

Hermann Suchier. Rudolf Kautzsch. 